\title{
Application of unsupervised pattern recognition approaches for exploration of rare earth elements in Se-Chahun iron ore, central Iran
}

\author{
Mohammadali Sarparandeh and Ardeshir Hezarkhani \\ Department of Mining and Metallurgical Engineering, Amirkabir University of Technology, Tehran, 9821, Iran
}

Correspondence: Ardeshir Hezarkhani (ardehez@aut.ac.ir)

Received: 16 June 2017 - Discussion started: 11 August 2017

Revised: 28 October 2017 - Accepted: 9 November 2017 - Published: 15 December 2017

\begin{abstract}
The use of efficient methods for data processing has always been of interest to researchers in the field of earth sciences. Pattern recognition techniques are appropriate methods for high-dimensional data such as geochemical data. Evaluation of the geochemical distribution of rare earth elements (REEs) requires the use of such methods. In particular, the multivariate nature of REE data makes them a good target for numerical analysis. The main subject of this paper is application of unsupervised pattern recognition approaches in evaluating geochemical distribution of REEs in the Kiruna type magnetite-apatite deposit of SeChahun. For this purpose, 42 bulk lithology samples were collected from the Se-Chahun iron ore deposit. In this study, 14 rare earth elements were measured with inductively coupled plasma mass spectrometry (ICP-MS). Pattern recognition makes it possible to evaluate the relations between the samples based on all these 14 features, simultaneously. In addition to providing easy solutions, discovery of the hidden information and relations of data samples is the advantage of these methods. Therefore, four clustering methods (unsupervised pattern recognition) - including a modified basic sequential algorithmic scheme (MBSAS), hierarchical (agglomerative) clustering, $k$-means clustering and selforganizing map (SOM) - were applied and results were evaluated using the silhouette criterion. Samples were clustered in four types. Finally, the results of this study were validated with geological facts and analysis results from, for example, scanning electron microscopy (SEM), X-ray diffraction (XRD), ICP-MS and optical mineralogy. The results of the $k$-means clustering and SOM methods have the best matches with reality, with experimental studies of samples and with field surveys. Since only the rare earth elements are used in
\end{abstract}

this division, a good agreement of the results with lithology is considerable. It is concluded that the combination of the proposed methods and geological studies leads to finding some hidden information, and this approach has the best results compared to using only one of them.

\section{Introduction}

In present study, the geochemical distribution of rare earth elements (REEs) was evaluated using bulk lithology samples for the first time in the Se-Chahun deposit. A clustering approach attempts to organize unlabeled feature vectors into clusters (natural groups) such that samples within a cluster are similar to each other but differ from those in other clusters (Hilario and Ivan, 2004). Clustering analysis is an important and useful tool for analyzing large datasets that contain many variables and experimental parameters. Therefore, the application of cluster analysis to complex datasets has attracted a high level of scientific interest in various aspects of geochemistry research (Nguyen et al., 2015). In order to investigate the distribution of elements, it is essential for a robust classification scheme to cluster chemistry samples into homogeneous groups (Guler et al., 2002). Several common clustering techniques have been utilized to divide geochemical samples into similar homogeneous groups with the ultimate objective of characterizing the quality of elements, such as principal component analysis, fuzzy $k$-means clustering technique and Q-mode hierarchical cluster analysis to assess the chemistry of groundwater and to identify the geological factors. For example, Ji et al. (2007) devel- 
oped semi-hierarchical correspondence cluster analysis and showed its application for division of geological units with the help of geochemical data that are systematically collected from an area around Tahe in Heilongjiang Province, north China. Meshkani et al. (2011) used hierarchical and $k$-means clustering for identifying distribution of lead and zinc in the Sanandaj-Sirjan metallogenic zone in Iran. Ziaii et al. (2009) introduced the neuro-fuzzy method for separating anomalies and showed that this method is more efficient than using multivariate statistics. Ellefsen and Smith (2016) evaluated a clustering method called the Bayesian finite mixture modeling procedure by applying it to geochemical data collected in the State of Colorado, United States of America.

The proposed method of the self-organizing maps (SOMs) is likely to become a complementary or an alternative tool to the clustering methods (Kalteh et al., 2008; Iseri et al., 2009). The SOM method is related to adaptive $k$-means method but performs a topological feature map that is more complex than just cluster analysis. After training, the input vectors are spatially ordered in the array; i.e., the neighboring input vectors on the map are more similar than the more remote ones (Du and Swamy, 2006). The self-organizing map approach is based on unsupervised learning algorithms and has excellent visualization capabilities including techniques that apply the reference vectors of the SOM to give an informative picture of the data (Lu et al., 2003). Sun et al. (2009) applied the SOM method to classify $\mathrm{Pb}-\mathrm{Zn}-\mathrm{Mo}-\mathrm{Ag}$ anomalies in the mining area around Sheduolong in Qinghai Province, China. In 2012, Abedi et al. the used the SOM and fuzzy $k$-means techniques to provide a deposit exploration map for the Now Chun copper deposit in Iran. Sarparandeh and Hezarkhani (2016) examined the application of SOMs in evaluation of the geochemical distribution of REEs in the Choghart $\mathrm{Fe}-$ REE deposit in Bafq district and showed its good performance. Generally, in cases where there are too many parameters and samples, pattern recognition is a suitable approach for data processing. Exploration of rare earth elements is one of these cases because of the multi-elemental nature of the data. For instance, in this study, 14 rare earth elements were measured with inductively coupled plasma mass spectrometry (ICP-MS). Pattern recognition makes it possible to evaluate the relations between the samples based on all these 14 features simultaneously. In addition to providing easy solutions, discovery of the hidden information and relations of data samples is the advantage of these methods. This paper suggests a new approach for exploration of REEs, which is more applicable and compatible with the multivariate nature of them.

\section{Geological settings of study area}

There are several deposits of iron ore in central and northeastern Iran, and magnetite is the main mineral in most of them. In most iron ore deposits of Iran, metasomatism is the main cause of concentration (NISCO, 1975). Systematic exploration work during the 1960s and 1970s outlined 34 zones of aeromagnetic anomalies between Bafq in the south and Saghand in the north with a total reserve of more than $1500 \mathrm{Mt}$ iron ore (Torab, 2008). The Se-Chahun deposit is composed of two major groups of ore bodies, called the $\mathrm{X}$ and XI anomalies (NISCO, 1975). Anomaly X crops out as some small black hills containing $11 \mathrm{Mt}$ iron ore reserve with mainly rich magnetite ore (Torab, 2008). Anomaly XI occurs $3 \mathrm{~km}$ northeast of anomaly $\mathrm{X}$. Each anomaly consists of two or three smaller tabular to lens-shaped ore bodies in association with other small bodies (Bonyadi et al., 2011). The mineralization is mainly hosted by metasomatized tuffs of andesite composition. A geological map of the Se-Chahun deposit (anomaly $\mathrm{X}$ ) and the location of samples within the study area are shown in Fig. 1.

\section{Mineralogy and lithology}

The host rocks have a gradual boundary. Samples mainly include iron ores, low-grade ores (transition zone, consisting of plagioclase and actinolite) and metasomatic rocks (mainly consisting of actinolite and plagioclase). Figure $7 \mathrm{a}$ and $\mathrm{c}$ show two examples of iron ores: phosphorus iron ore with large amounts of REEs (Fig. 7a) and iron ore with small amounts of REEs (Fig. 7c). Apatites can be seen in hand specimens by the cream-pink color (Fig. 7a). Some examples for metasomatic host rock are presented in Fig. 7b and d. They are mainly pale green. The main minerals in host rocks are shown in microscopic images. The ore body is comprised of high-grade magnetite. The most important REEbearing minerals in the Se-Chahun deposit are apatite and monazite. There are two types of apatite: REE-bearing apatite and depleted apatite. Bonyadi et al. (2011) showed that some apatites of Se-Chahun have been leached of light REEs (LREEs), $\mathrm{Y}, \mathrm{Na}, \mathrm{Cl}, \mathrm{Mg}, \mathrm{Mn}$ and $\mathrm{Fe}$. REE-bearing apatites are bright in back-scattered electron (BSE) images, while leached apatites are dark. In terms of dimensions, there are two types of apatite: coarse grain and fine grain. They can be seen under optical and scanning electron microscopes. However, all of them are extremely altered, and their crystals can not be seen in hand samples. The content of rare earth elements is directly related to the amount of apatite. The more the apatite, the greater the amount of REEs. Monazites are very fine grains and can only be distinguished in scanning electron microscopy (SEM) images (Fig. 2). They are brighter than apatites and magnetites and contain greater amounts of REEs. However, there are small amounts of monazite in samples. Therefore, apatite is the main source of REEs in the Se-Chahun deposit. However, in cases with medium amounts of REEs, it is found that there is a different condition. In fact, there is another group of samples in which there are lesser amounts of $\mathrm{P}$ with considerable concentrations of REEs. This group of data was separated easily 


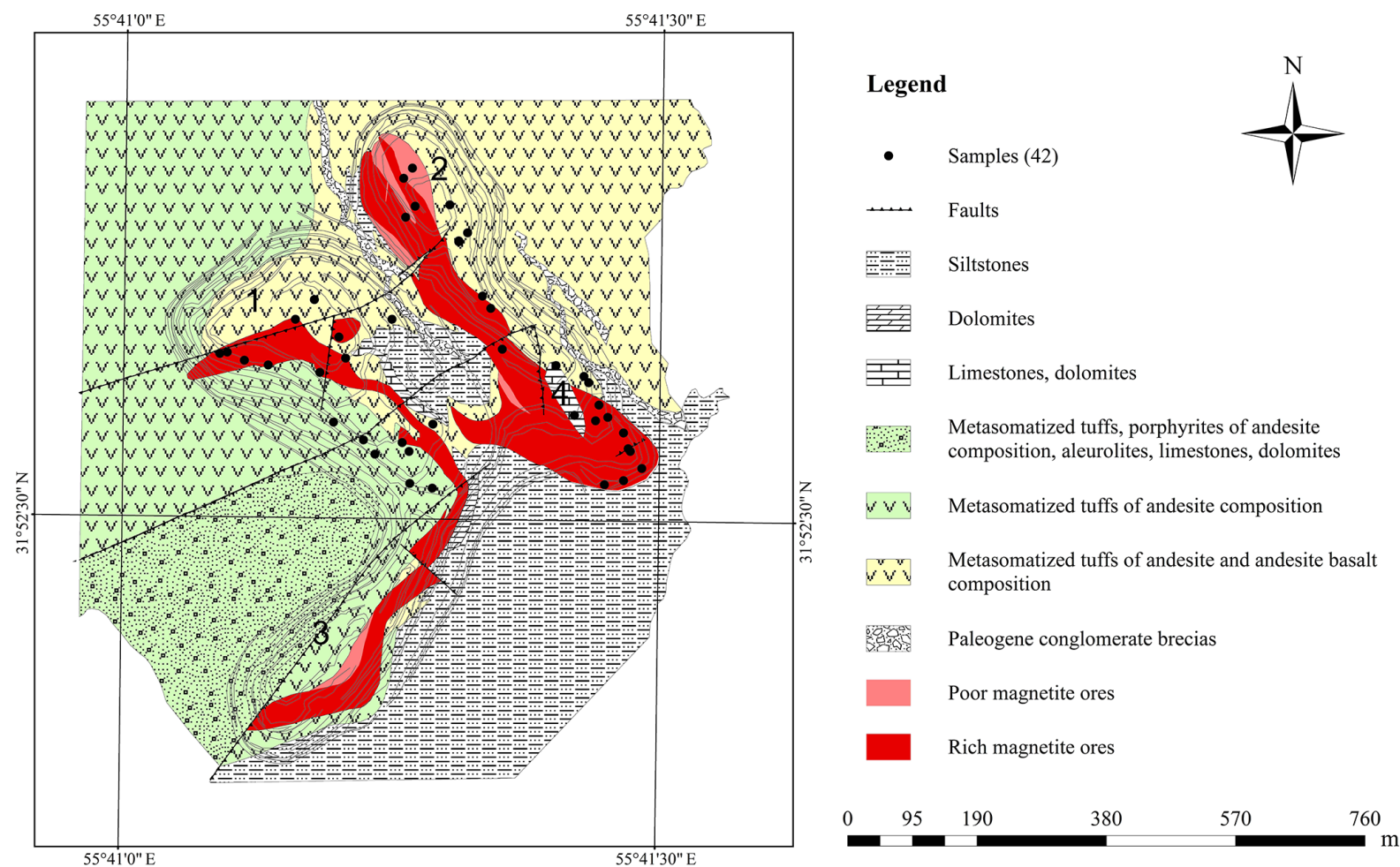

Figure 1. Geological map of Se-Chahun deposit (anomaly X) and sample locations. Contours of open pits are shown on the map, and the open pits are numbered from 1 to 4 (supplementary part of pit 2 is known as pit 4) (modified after NISCO, 1975).
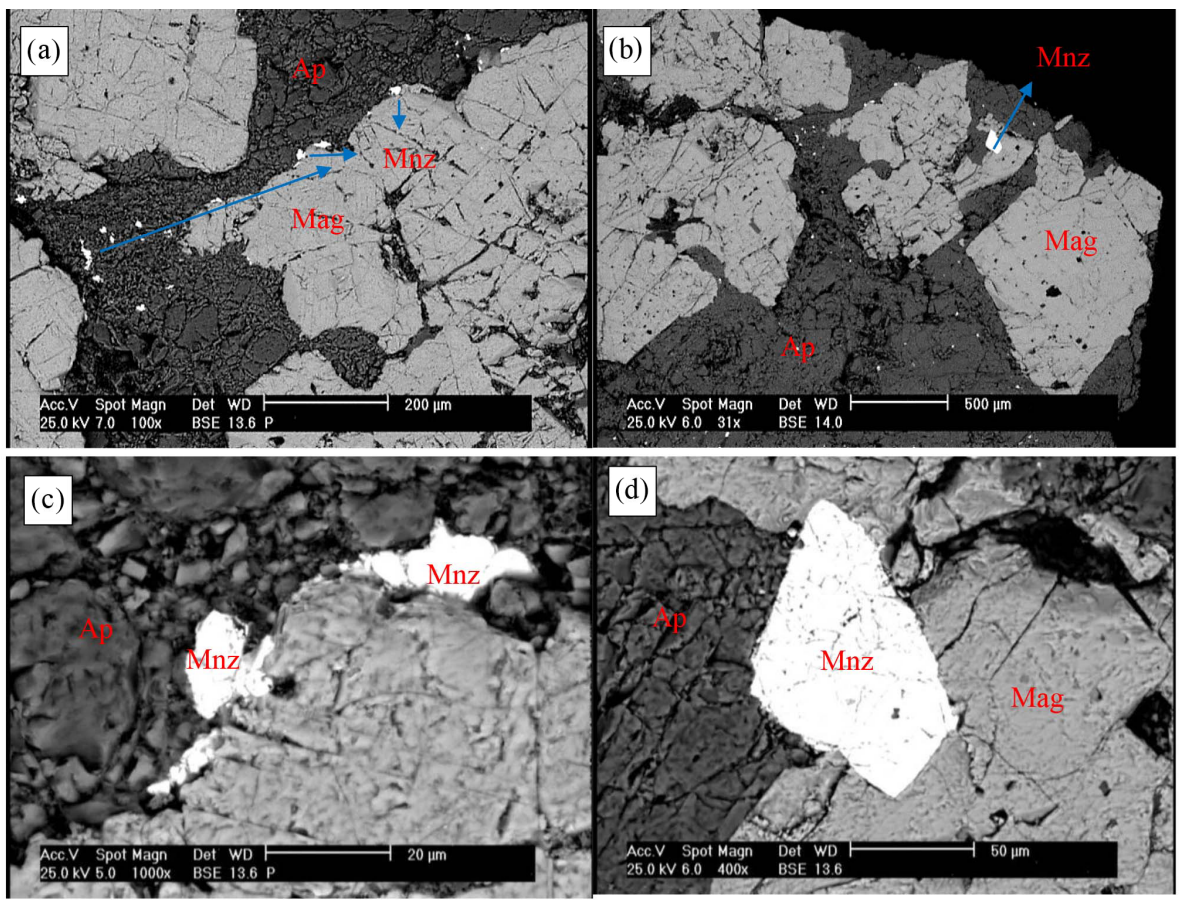

Figure 2. Back-scattered electron images of a sample from phosphate rocks. Abbreviations: Ap - apatite; Mnz - monazite; and Mag magnetite. 


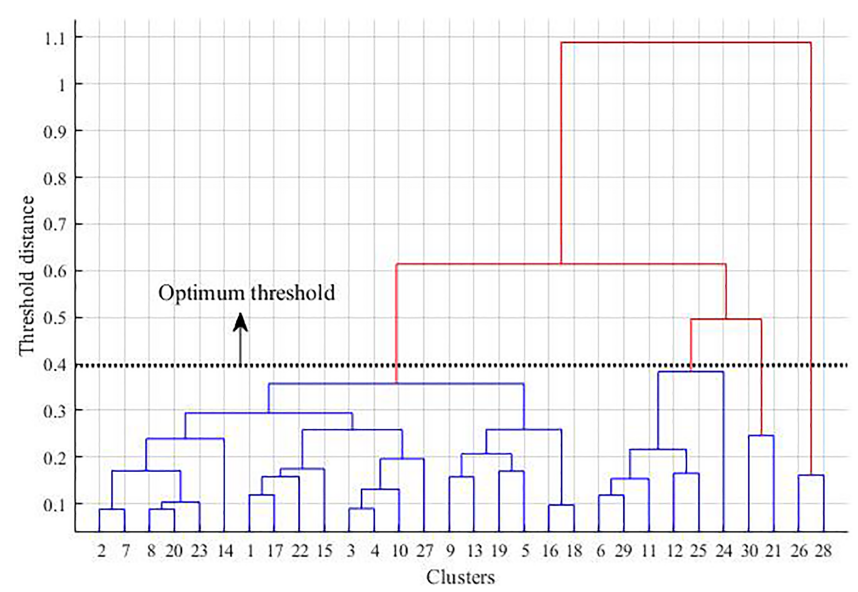

Figure 3. Dendrogram for identifying the optimum threshold and number of clusters.

by clustering methods. This was confirmed by evaluation of samples under SEM. After a complete survey of samples under SEM, it was found that the samples of this cluster (e.g., Fig. 7b) contain monazite with an absence of apatite.

\section{Scanning electron microscopy}

Several samples were analyzed with SEM, and the results were used for evaluation of mineralogy and also validity of this study. Figure 2 shows the BSE images of a sample from phosphate rocks. Monazites are brightly colored and include $\mathrm{Ce}, \mathrm{La}$ and $\mathrm{Nd}$. Apatites are dark gray and include $\mathrm{P}, \mathrm{Ca}$ and La but no Ce. As can be seen in Fig. 2, there are small amounts of monazite. Monazites can be seen in two ways: (1) small crystals around the apatite and (2) inclusions in apatite crystals (Fig. 2a).

\section{Chemical analysis}

In this study, 42 bulk lithology samples were collected from anomaly X of Se-Chahun iron ore deposits. They are from pit 1, 2 and 4 (supplementary part of pit 2 is known as pit 4 , Fig. 1). 19 samples were taken from pit 1,9 samples from pit 2 and 14 samples from pit 4 . Samples were taken from the ore body and metasomatic zones. After preparation of the samples, they were analyzed with ICP-MS. The concentrations of REEs were normalized between 0 and 1 and were used as input data for clustering. These data can be divided roughly into three groups: samples with high, medium and low concentration of REEs. Accurate determination of groups requires multivariate analysis and data processing. Another important point is that the samples are enriched by LREEs and Y. Large amounts of REEs occur in phosphorus iron ores, and they are more in the supplementary part of pit 2 (or pit 4). Assayed REEs are 14 elements: $\mathrm{La}, \mathrm{Ce}, \mathrm{Pr}, \mathrm{Nd}, \mathrm{Sm}, \mathrm{Eu}$,
Gd, Tb, Dy, Er, Tm, Yb, Lu and Y. Mean, variance, minimum and maximum of these rare earth elements are presented in Table 1.

\section{Methodology}

Four methods - a modified basic sequential algorithmic scheme (MBSAS), hierarchical (agglomerative) clustering, $k$-means clustering and SOM - were applied in this study. These methods have been applied in diverse aspects of science and engineering, somewhat in geochemistry and never for exploration of REEs. The papers of Sarparandeh and Hezarkhani (2016) and Zaremotlagh and Hezarkhani (2016) are the only efforts which have been made in this area. However, there is no study that applies and compares several types of algorithms. In this study, in addition to providing such useful information and experience, the authors show that some extra information such as the relation between REEs content and lithology of samples can be achieved by the proposed methods. Moreover, a good discrimination based on lithology is attained just by using REEs. The general concepts of each method are explained in the following.

\subsection{Sequential clustering}

Sequential methods are easy and fast algorithms. These include a basic sequential algorithmic scheme (BSAS) as well as a modifed version (MBSAS). In BSAS two parameters should be defined by the user: the maximum number of clusters and dissimilarity threshold. The basic idea behind BSAS is that each input vector $\boldsymbol{x}$ is assigned to an already created cluster or a new one is formed. Therefore, a decision for vector $\boldsymbol{x}$ is reached prior to the final cluster formation, which is determined after all vectors have been presented. The refinement of BSAS, which is called modified BSAS (MBSAS), overcomes this drawback. The algorithmic scheme consists of two phases. The first phase involves the determination of the clusters, via the assignment of some of the vectors of $\boldsymbol{x}$ to them. During the second phase, the unassigned vectors are presented for a second time to the algorithm and are assigned to the appropriate cluster (Theodoridis and Koutroumbos, 2003). Therefore, in this study the MBSAS algorithm was applied for clustering of samples based on REEs. In this study, the mean of each group and the Euclidean distance were used as the cluster centers and a measure of dissimilarity, respectively.

\subsection{Hierarchical clustering}

Hierarchical clustering procedures are among the most commonly used methods of summarizing data structure. They use a hierarchical tree, which is a nested set of partitions represented by a tree diagram or dendrogram (Fig. 3). To separate each branch of the dendrogram, a numerical value that indicates the dissimilarity between clusters should be measured. 

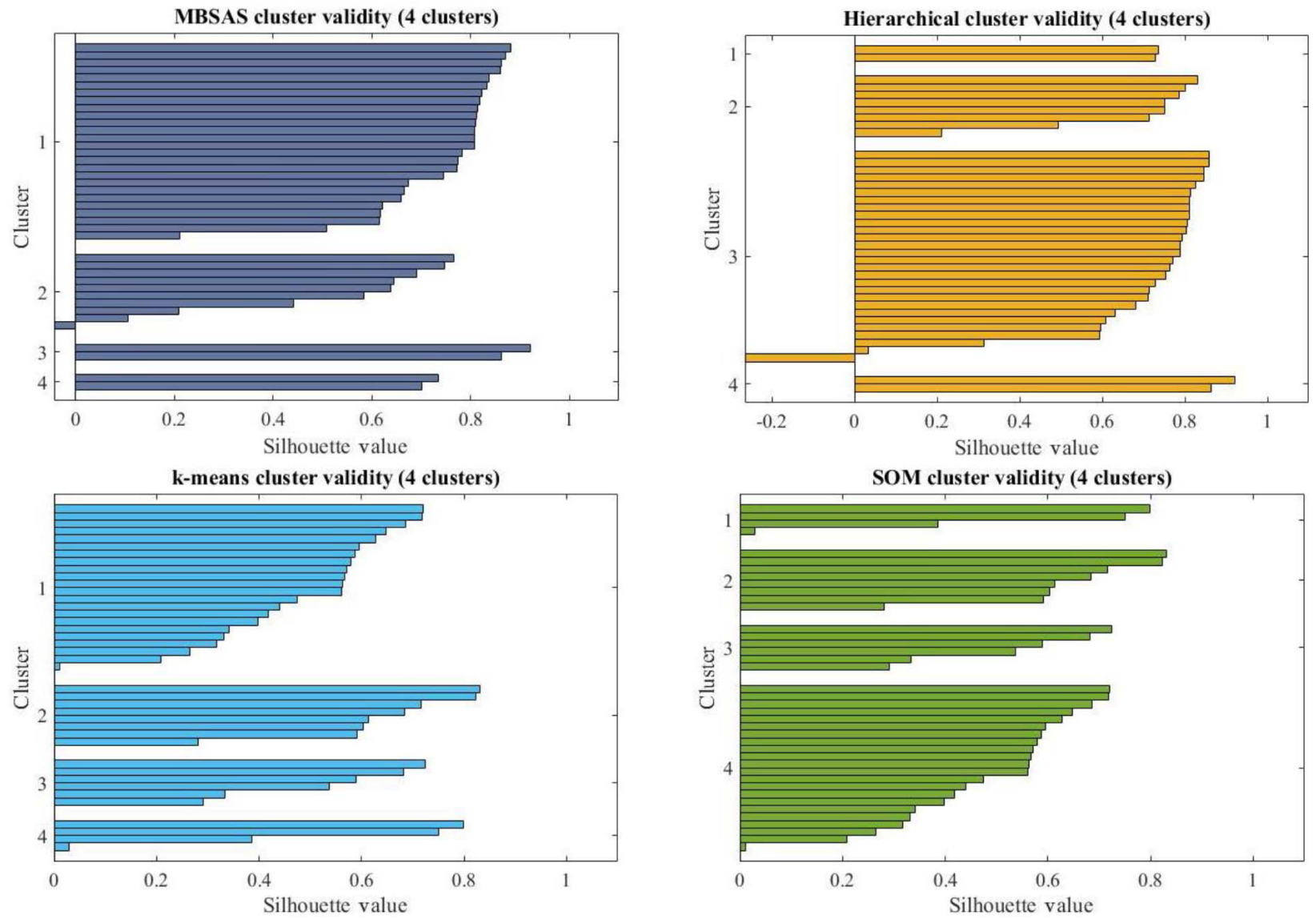

Figure 4. Silhouette plots for each method show the validity of each sample in a certain cluster. Positive values show that the sample has been clustered in the correct group and its magnitude is a measure of accuracy.

Table 1. Mean, variance, minimum and maximum of 14 assayed rare earth elements in 42 samples.

\begin{tabular}{lrrrrrrrrrrrrrr}
\hline Elements (ppm) & $\mathrm{La}$ & $\mathrm{Ce}$ & $\mathrm{Pr}$ & $\mathrm{Nd}$ & $\mathrm{Sm}$ & $\mathrm{Eu}$ & $\mathrm{Gd}$ & $\mathrm{Tb}$ & $\mathrm{Dy}$ & $\mathrm{Er}$ & $\mathrm{Tm}$ & $\mathrm{Yb}$ & $\mathrm{Lu}$ & $\mathrm{Y}$ \\
\hline Mean & 73 & 154 & 20 & 75 & 13 & 2 & 13 & 2 & 12 & 7 & 1 & 13 & 1 & 56 \\
Variance & 27180 & 111800 & 1202 & 15180 & 299 & 3 & 242 & 4 & 121 & 40 & 1 & 64 & 0 & 2869 \\
Minimum & 3 & 2 & 0 & 5 & 1 & 0 & 1 & 0 & 1 & 1 & 0 & 1 & 0 & 9 \\
Maximum & 995 & 2037 & 203 & 740 & 102 & 9 & 90 & 12 & 60 & 32 & 4 & 42 & 3 & 305 \\
\hline
\end{tabular}

There are several different algorithms for finding a hierarchical tree. An agglomerative algorithm begins with $\mathrm{n}$ subclusters, each containing a single data point, and at each stage merges the two most similar groups to form a new cluster, thus reducing the number of clusters by one. The algorithm proceeds until all the data fall within a single cluster. A divisive algorithm operates by successively splitting groups, beginning with a single group and continuing until there are $n$ groups, each of a single individual. Generally, divisive algorithms are computationally inefficient, except where most of the variables are binary attribute variables (Webb, 2002). In this study, an agglomerative approach was used.

\section{3 $K$-means clustering}

$K$ means is one of the most popular and well-known clustering algorithms. In this method, first, $k$ samples are considered as initial cluster centers. Then, distances between the points and these centers are calculated, and the nearest points to each center are assigned to that cluster. Next, the mean of each cluster will be used as a new center. This process continues until no changes appear in the clusters (Theodoridis and Koutroumbos, 2003). The $k$-means algorithm seeks to partition the data into $k$ groups or clusters so that the within-group sum of squares is minimized (Webb, 2002). 

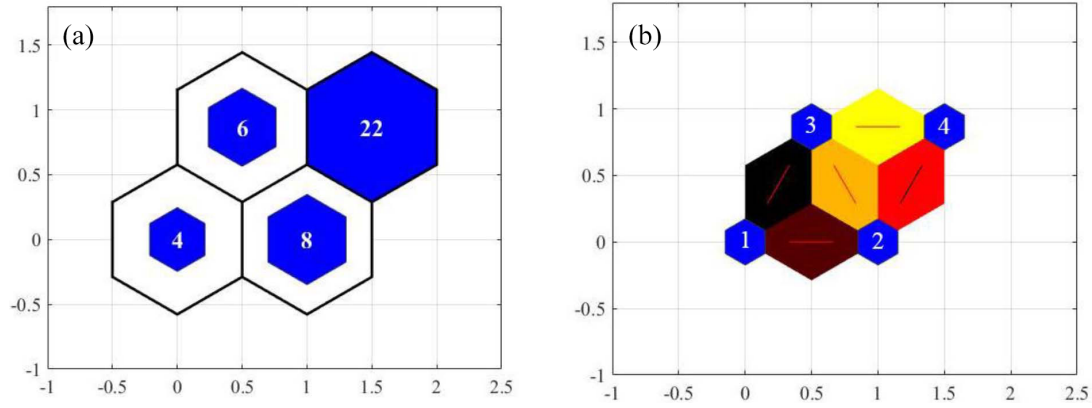

Figure 5. SOM topology and determining the number of samples for each cluster (a), SOM neighbor weight distances and neighbor connections (b).

MBSAS

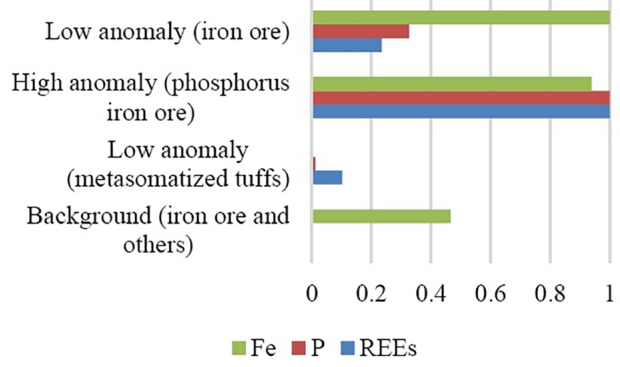

K-means

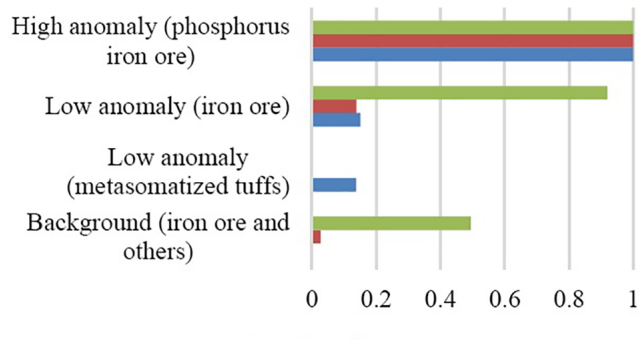

$\square \mathrm{Fe} \backsim \mathrm{P} \backsim \mathrm{REES}$
Hierarchical

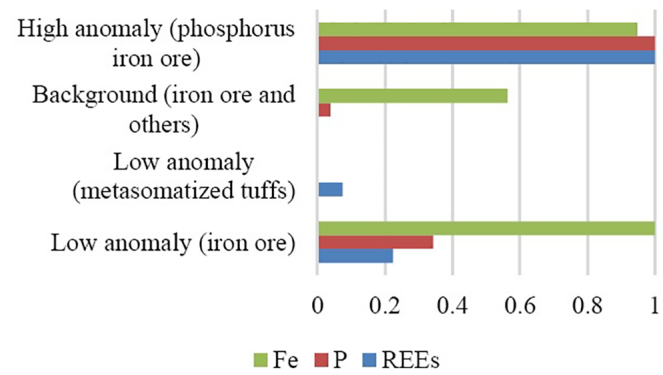

SOM

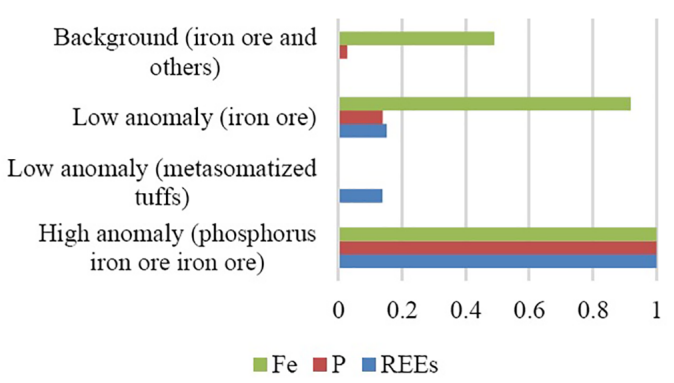

Figure 6. Comparative bar charts of normalized values of REE, $\mathrm{P}$ and $\mathrm{Fe}$ for all clustering methods.

\subsection{Self-organizing map}

An SOM is a kind of artificial neural network (ANN). It can be used for unsupervised clustering. This method was introduced by Kohonen in 1980, and their main application is to reduce the dimensional (Kohonen, 1998). In this method, topological structure of the input space will be saved. The net of neurons can be a right angle or hexagonal grid, and the adjacent cells upgrade during successive stages (Engelbrecht, 2002).

\subsection{Cluster validity}

The optimum number of clusters was found by the silhouette method. In this method, a graphical validation was used for evaluating the number of clusters and comparing different scenarios. Therefore, by calculating the distances between samples in the clusters and distances between the prototypes the optimal number was determined (Rousseeuw, 1987).

\section{Results and discussion}

The aim of this study is to investigate the geochemical distribution of REEs. Therefore, the concentrations of REEs (after normalization between 0 and 1) were used as input data for clustering. But, after data processing, the clustering results were compared with concentrations of phosphorus and iron. Moreover, the lithology of samples was considered for validation. Clustering results of four methods - MBSAS, hierarchical (agglomerative) clustering, $k$-means clustering and $\mathrm{SOM}-$ will be discussed in the following. 
(a)
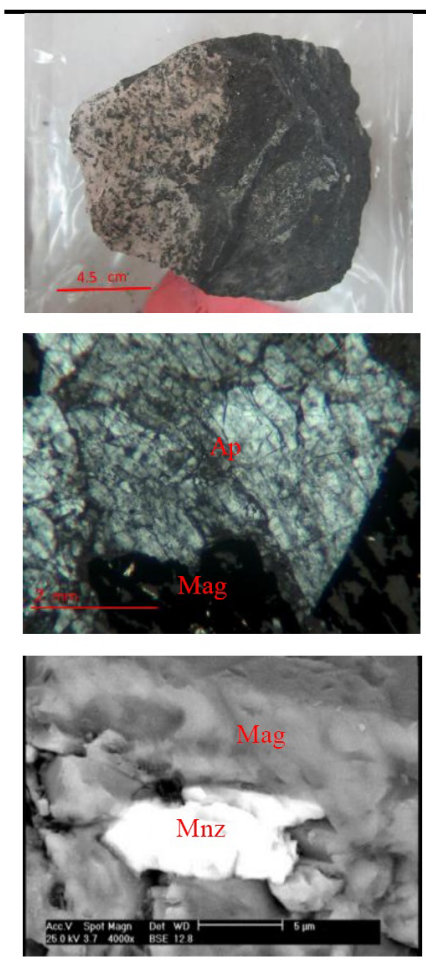

(b)
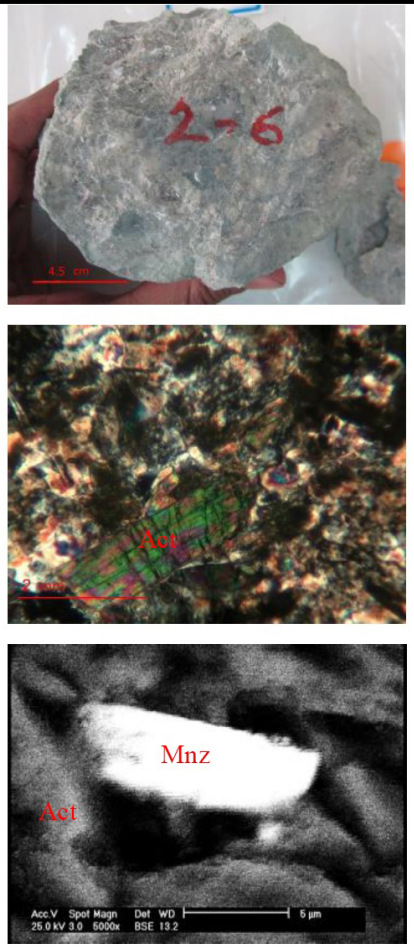

(c)
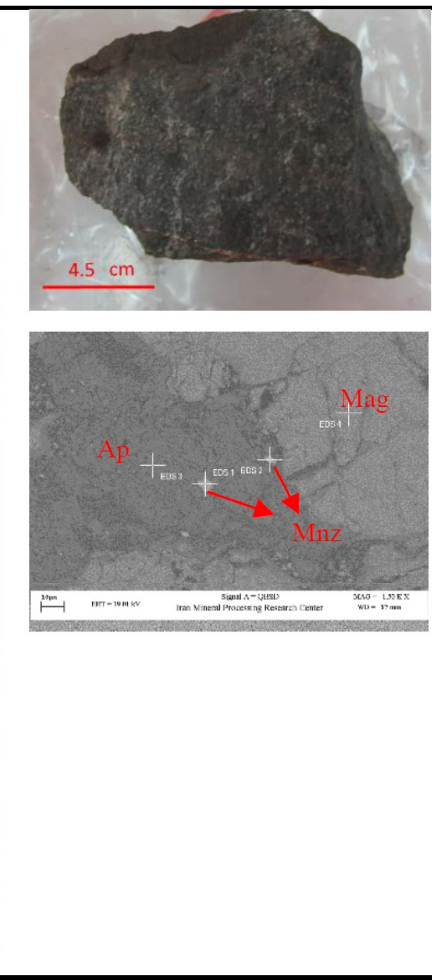

Figure 7. Some examples for samples which have been classified in four types along with their microscopic images. (a) Type 1: high anomaly (phosphorus iron ore), sample 4-1, iron ore sample including apatite and monazite; (b) type 2: low anomaly (metasomatized tuffs), sample 2-6, including actinolite, calcite, feldspar and monazite; (c) type 3, low anomaly (iron ore), sample 4-6, iron ore sample including apatite (mostly depleted from REEs) and monazite; (d) type 4: background (iron ore and others), sample 1-16, metasomatite including plagioclase, feldspar and actinolite. Abbreviations: Ap - apatite; Mnz - monazite; Act - actinolite; Mag - magnetite; and Pl - plagioclase.

The input of the methods is a dataset of 42 vectors with 14 dimensions (42 samples and 14 rare earth elements). First, outliers should be put aside. For this purpose, the dendrogram based on the average of each cluster and Euclidean distance between the clusters was composed. Linkage analysis showed that two samples have more distance from others and can be put aside as outliers. They are phosphorous iron ore with high concentrations of REEs. Contents of REEs in these two samples are much higher than in others. They belonged to certain clusters (due to the similarity) at the end of calculations.

In MBSAS and hierarchical methods, two parameters (i.e., optimum threshold and number of clusters) should be identified. To this end, the dendrogram was drawn. Figure 3 shows the dendrogram for identifying the optimum threshold and number of clusters. It has been calculated based on the average of each cluster and Euclidean distance between the clusters. The optimum threshold was identified as 0.4 based on the endrogram (Fig. 3). In this way, four clusters were obtained. However, for all four methods, the number of clusters was changed in the range of 2-6, and then results were evaluated using the silhouette criterion. Finally, four clusters was decided upon as the optimal number. In this case the best results of silhouette values were attained for all methods. Silhouette plots for each method show the validity of each sample in a certain cluster. Positive values show that the sample has been clustered in the correct group and its magnitude is a measure of accuracy. Results of the silhouette method are shown in Fig. 4. As can be seen in Fig. 4, one sample in the MBSAS and hierarchical methods has a negative value. This means that this sample is in the wrong cluster. Comparing the results of the methods shows that the MBSAS and hierarchical methods had the same outputs, and so the $k$-means and SOM methods have similar outputs. Moreover, results of the $k$-means and SOM methods have the best matches with reality, with experimental studies of samples and with field surveys.

Characteristics of each cluster in each method are summarized in Table 2. For this purpose, averages of $\sum$ REEs (total concentrations of rare earth elements) as well as $\mathrm{P}$ and Fe for each cluster have been calculated. Comparing these results with laboratory analyses and field studies, we concluded that samples can be classified into four types (Fig. 7): (1) high anomaly (phosphorus iron ore), (2) low anomaly (metasom- 
Table 2. Characteristics of each cluster in each method. Iron and phosphorus concentrations are shown for comparison.

\begin{tabular}{|c|c|c|c|c|}
\hline \multicolumn{5}{|c|}{ MBSAS } \\
\hline Cluster no. & $\sum$ REEs (ppm) & $\mathrm{P}(\mathrm{ppm})$ & $\mathrm{Fe} \%$ & Description \\
\hline 1 & 169 & 933 & 29 & Background (iron ore and others) \\
\hline 2 & 422 & 1294 & 10 & Low anomaly (metasomatized tuffs) \\
\hline 3 & 2646 & 31934 & 47 & High anomaly (phosphorus iron ore) \\
\hline 4 & 749.14 & 11061 & 49 & Low anomaly (iron ore) \\
\hline \multicolumn{5}{|c|}{ Hierarchical } \\
\hline Cluster no. & $\sum$ REEs (ppm) & $\mathrm{P}(\mathrm{ppm})$ & $\mathrm{Fe} \%$ & Description \\
\hline 1 & 749 & 11061 & 49 & Low anomaly (iron ore) \\
\hline 2 & 383 & 123 & 4 & Low anomaly (metasomatized tuffs) \\
\hline 3 & 199 & 1335 & 29 & Background (iron ore and others) \\
\hline 4 & 2646 & 31934 & 47 & High anomaly (phosphorus iron ore) \\
\hline \multicolumn{5}{|c|}{$k$ means } \\
\hline Cluster no. & $\sum$ REEs (ppm) & $\mathrm{P}(\mathrm{ppm})$ & $\mathrm{Fe} \%$ & Description \\
\hline 1 & 143 & 775 & 25 & Background (iron ore and others) \\
\hline 2 & 383 & 143 & 3 & Low anomaly (metasomatized tuffs) \\
\hline 3 & 407 & 3387 & 44 & Low anomaly (iron ore) \\
\hline 4 & 1887 & 23585 & 48 & High anomaly (phosphorus iron ore) \\
\hline \multicolumn{5}{|c|}{ SOM } \\
\hline Cluster no. & $\sum$ REEs (ppm) & $\mathrm{P}(\mathrm{ppm})$ & $\mathrm{Fe} \%$ & Description \\
\hline 1 & 1887 & 23585 & 48 & High anomaly (phosphorus iron ore iron ore) \\
\hline 2 & 383 & 123 & 4 & Low anomaly (metasomatized tuffs) \\
\hline 3 & 407 & 3387 & 44 & Low anomaly (iron ore) \\
\hline 4 & 143 & 775 & 25 & Background (iron ore and others) \\
\hline
\end{tabular}

Table 3. Concentrations of REEs (ppm), P (ppm) and Fe (\%) for the samples of Fig. 7.

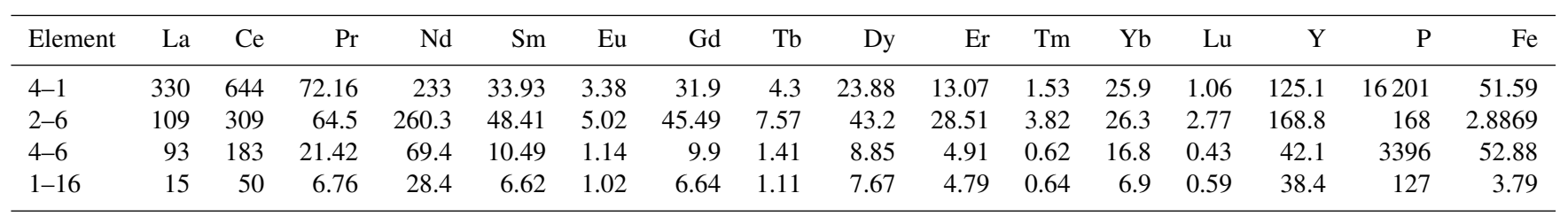

atized tuffs), (3) low anomaly (iron ore), and (4) background (iron ore and others). Since only the rare earth elements are used in this division, a good agreement of the results with lithology is considerable. Type 1 is comprised of iron ore with a high anomaly of REEs (about 1900 ppm) and the high content of phosphorus (more than $2 \%$ ). Figure 2 shows SEM images of a sample from type 1 . This type is the most prone to rare earth elements and containing apatite and monazite. However, fluorapatite is the main mineral of REEs in this type (due to the X-ray diffraction (XRD) and SEM analyses). The second type (i.e., metasomatized tuffs) has a low anomaly of REEs, whereas the concentration of $\mathrm{P}$ is low. Samples of this group are metasomatized tuffs of andesite composition and mainly consist of actinolite and plagioclase with low concentrations of Fe and $\mathrm{P}$, but the contents of REEs are considerable (on average about $400 \mathrm{ppm}$ ). SEM analysis shows that monazite is the mineral of REEs and apatite does not exist in this type. The third type shows a low anomaly of REEs with the lithology of the ore body and relatively high content of P (about $3400 \mathrm{ppm}$ in SOM and $k$-means results and about $1 \%$ in MBSAS and hierarchical clustering). The last type is background (low concentrations of REEs) and comprised of various samples of iron ore and others (mainly metasomatic samples).

As mentioned above, the results of the $k$-means clustering and SOM methods have the best matches with reality, with experimental studies of samples and with field surveys. However, a self-organizing map has the capability to 
present a two-dimensional map (for visual evaluation of clusters) from multidimensional data. In addition, the weight distance matrix provides a tool to compare clusters. These advantages of the SOM method make it more applicable for data processing in exploration works. Figure 5a shows the SOM topology which has been used in this study as well as the number of samples for each cluster. Since a SOM has a two-dimensional topology, the relations between centers of 14-dimensional clusters have been illustrated on a twodimensional map. Weight distance matrix or unified distance matrix (U matrix) is one of the SOM's tools. Figure 5b shows neighbor weight distances. Lines are used to display the relationship between neighboring neurons. The darker the color, the greater the distance between the neurons; the lighter the color, the smaller the distance between the neurons. Therefore, as can be seen in Fig. 5b, type 1 (i.e., high anomaly or phosphorus iron ore) has the maximum distance with type 3 and to a lesser degree with type 2. Also, type 3 and 4 are closest together and most similar to each other in terms of REEs. Finally, type 1 or phosphorus iron ore type is the most promising type for rare earth elements. This type occurs mainly in the supplementary part of pit 2 (or pit 4). For a better comparison of the four methods, the outputs of clustering algorithms (Table 2) were normalized, and the results were summarized in four bar charts (Fig. 6).

In this study, pattern recognition helped to divide the samples into appropriate groups, according to the contents of REEs, and results are consistent with the concentration of $\mathrm{P}$ and with the lithology of the samples. The variety of parameters, especially in case of REE explorations, somewhat complicates for interpretation of the data and exploration area. Since single-variable methods do not provide useful information, the authors proposed four common clustering algorithms, which have been explained above. The output of these four methods (Fig. 6 and Table 2) shows that the discrimination of clusters is based on the lithology of the samples, in addition to the REEs. Therefore, it is proven that proposed methods have found the relation between the distribution of REEs and the lithology of the study area. In this regard, we claim that pattern recognition helps to find some hidden information associated with the complicated nature of REE systems. Figure 7 is prepared to show the application and efficiency of unsupervised methods in evaluating geochemical distribution of REEs in the Kiruna type magnetiteapatite deposit of Se-Chahun, while it does not need to do additional geological studies with extra cost and time. These samples are shown as the representative samples for each cluster. Their contents of REEs are presented in Table 3. Sample 4-1 (phosphorus iron ore, Fig. 7a) contains about $9 \%$ apatite (based on XRD analysis). There are high contents of REEs $\left(\sum\right.$ REEs $\left.=1543 \mathrm{ppm}\right)$ and $\mathrm{P}(16201 \mathrm{ppm})$ in this sample. Figure $7 b$ is an example (sample no. 2-6) for type 2 (low anomaly, metasomatized tuffs). Rare earth elements of this type are from monazite. Apatite was not observed in this type, and therefore the concentration of $\mathrm{P}$ is relatively lower. BSE image of a monazite in sample 2-6 is shown in Fig. 7b. Figure 7c is a sample (4-6) for iron ore with a low anomaly of REEs. Apatites of this sample are mainly depleted from REEs. They were observed under SEM (depleted apatites are darker in BSE images). Therefore, although there are large amounts of $\mathrm{P}$ in it, the concentrations of REEs are relatively lower (Table 3). A metasomatite sample (nos. 1-16) is shown in Fig. 7d as an example for background. Plagioclase and actinolite are the main minerals of it. Concentrations of $\sum$ REEs and $\mathrm{P}$ in this sample are 175 and $127 \mathrm{ppm}$, respectively.

Four methods - MBSAS, hierarchical (agglomerative) clustering, $k$-means clustering and SOM - were applied in this study. However, the $k$-means clustering and SOM methods are more advanced in comparison to others. They improve and modify the weights or centers of the clusters continuously in several stages. In contrast, the MBSAS and hierarchical methods are more simple and elementary, because the centers of clusters are determined in one stage. Furthermore, SOM has the advantage that the distances between the clusters can be assessed visually on a two-dimensional map (Fig. 5b). Since the input dataset is comprised of 14dimensional vectors (14 REEs), SOM is a good tool for evaluating it in a two-dimensional space.

\section{Conclusions}

The following points were concluded:

- Successful clustering of a dataset which is consistent with geological facts and laboratory and field studies was achieved.

- The results of the $k$-means clustering and SOM methods have the best matches with reality, with experimental studies of samples and with field surveys.

- Since only the rare earth elements were used in this division, a good agreement of the results with lithology is considerable.

- Results showed that the unsupervised pattern recognition helps to find some hidden informations, which would be difficult to achieve in usual ways (i.e., finding the appropriate clusters). Methods which have been presented in this study will help better interpretation of data, despite there being many variables.

- A combination of numerical models and geological studies leads to the best outputs and outcomes in exploration programs of REEs.

- The proposed methods help to reduce the time and cost by eliminating the need for additional geological studies. 
Data availability. Data can be obtained on request from the authors.

Competing interests. The authors declare that they have no conflict of interest.

Acknowledgements. We thank the Iranian Mines and Mining Industries Development and Renovation Organization (IMIDRO) for financial support. We also thank the Iran Central Iron Ore Company and the personnel of the mine for their cooperation and contributions regarding mine visitations, sampling and field operations.

Edited by: Luis Vazquez

Reviewed by: Amir Mollajan and one anonymous referee

\section{References}

Abedi, M., Norouzi, G. H., and Torabi, S. A.: Clustering of mineral prospectivity area as an unsupervised classification approach to explore copper deposit, Arab. J. Geosci., 10, 3601-3613, 2012.

Bonyadi, Z., Davidson, G. J. Mehrabi, B., Meffre, S., and Ghazban, F.: Significance of apatite REE depletion and monazite inclusions in the brecciated Se-Chahun iron oxide apatite deposit, Bafq district, Iran: insights from para-genesis and geochemistry, Chem. Geol., 281, 253-269, 2011.

Du, K. L. and Swamy, M. N. S.: Neural Networks in a Softcomputing Framework, Springer-Verlag, London, 194, 2006.

Ellefsen, K. J. and Smith, D. B.: Manual hierarchical clustering of regional geochemical data using a Bayesian finite mixture model, Appl. Geochem., 75, 200-210, https://doi.org/10.1016/j.apgeochem.2016.05.016, 2016.

Engelbrecht, A., P.: Computational Intelligence, Wiley, Chichester, 63-73, 2002.

Guler, C., Thyne, G. D., McCray, J. E., and Turner, K. A.: Evaluation of graphical and multivariate Statistical methods for classification of water chemistry data, Hydrogeol. J., 10, 455-474, 2002.

Hilario, L. G. and Ivan, M. G.: Self-organizing map and clustering for wastewater treatment monitoring, Eng. Appl. Artif. Intel., 17, 215-225, 2004.

Iseri, E., Matsuura, T., Iizuka, S., Nishiyama, K., and Jinno, K.: Comparison of pattern extraction capability between selforganizing maps and principal component analysis, Memoirs of the Faculty of Engineering, Kyushu University, 69, 37-47, 2009.
Ji, H., Zeng, D., Shi, Y., Wu, Y., and Wu, X.: Semi-hierarchical correspondence cluster analysis and regional geochemical pattern recognition, J. Geochem. Explor., 93, 109-119, 2007.

Kalteh, A. M., Hjorth, P., and Berndtsson, R.: Review of the selforganizing map (SOM) approach in water resources: analysis, modeling and application, Environ. Modell. Softw., 23, 835-845, 2008.

Kohonen, T.: The self-organizing map, Neurocomputing, 21, 1-6, 1998.

Lu, H., Wu, Y., and Chen, S.: A new method based on SOM network to generate coarse meshes for overlapping unstructured multigrid algorithm, Appl. Math. Comput., 140, 353-360, 2003

Meshkani, S. A., Mehrabi, B., Yaghubpur, A., and Alghalandis, Y. F.: The application of geochemical pattern recognition to regional prospecting: a case study of the sanandaj-sirjan metallogenic zone, Iran, J. Geochem. Explor., 108, 183-195, 2011.

National Iranian Steel Corporation: Report on detailed exploration of Se-Chahun iron ore deposit in central Iran, National Iranian Steel Corporation (NISCO), Tehran, 1975.

Nguyen, T. T., Kawamura, A., Tong, T. N., Naoko, N., Hideo, A., and Romeo, G.: Clustering spatio-seasonal hydrogeochemical data using self-organizing maps for groundwater quality assessment in the Red river delta, Vietnam, J. Hydrol., 522, 661-673, 2015.

Rousseeuw, P. J.: Silhouettes: a graphical aid to the interpretation and validation of cluster analysis, J. Comput. Appl. Math., 20, 53-65, 1987.

Sarparandeh, M. and Hezarkhani, A.: Application of self-organizing map for exploration of REEs' deposition, Open J. Geol., 6, 571582, 2016.

Sun, X., Deng, J., Gong, Q., Wang, Q., Yang, L., and Zhao, Z.: Kohonen neural network and factor analysis based approach to geochemical data pattern recognition, J. Geochem. Explor., 103, 6-16, 2009.

Theodoridis, S. and Koutroumbos, K.: Pattern Recognition, Elsevier Academic Press, San Diego, 2003.

Torab, F.: Geochemistry and metallogeny of magnetite apatite deposits of the Bafq mining district, Central Iran, Dissertation, Clausthal University of Technology, Germany, 2008.

Webb, A. R.: Statistical Pattern Recognition, John Wiley \& Sons Ltd., England, 2002.

Zaremotlagh, S. and Hezarkhani, A.: A geochemical modeling to predict the different concentrations of REE and their hidden patterns using several supervised learning methods: choghart iron deposit, Bafq, Iran, J. Geochem. Explor., 165, 35-48, 2016.

Ziaii, M., Pouyan, A. A., and Ziaii, M.: Neuro-fuzzy modelling in mining geochemistry: identification of geochemical anomalies, J. Geochem. Explor., 100, 25-36, 2009. 